\title{
THRESHOLD BEHAVIOUR OF SIR EPIDEMIC MODEL WITH AGE STRUCTURE AND IMMIGRATION.
}

\author{
ANDREA FRANCESCHETTI AND ANDREA PUGLIESE
}

\begin{abstract}
We consider a SIR age-structured model with immigration of infectives in all epidemiological compartments; the population is supposed in demographic equilibrium between below-replacement fertility and immigration; the spread of the infection occurs through a general age-dependent kernel. We analyse the equations for steady states; because of immigration of infectives a steady state with a positive density of infectives always exists; however, a quasi-threshold theorem is proved, in the sense that, below the threshold, the density of infectives is close to 0 , while it is away from 0 , above the threshold; furthermore, conditions that guarantee uniqueness of steady states are obtained. Finally, we present some numerical examples, inspired to the Italian demographic situation, that illustrate the threshold-like behaviour, and other features of the stationary solutions and of the transient.
\end{abstract}

\section{INTRODUCTION.}

The current demographic trend in many Western countries is characterized by transition to sustained below replacement fertility and a sizeable immigration[16, 25]. Among the many aspects influenced by this major transition, it has been surmised that the dynamics of childhood infectious diseases (such as measles) could be substantially affected [20]). Understanding the possible consequences of such a transition with the help of mathematical models is important in planning public health policies, for example vaccination strategies aimed at the control and elimination of an infectious disease in a population [18].

In this paper we analyse a SIR age-structured model for the spread of an infectious disease in a population subject to below replacement fertility and immigration. The model is built, on the one hand, on the stable population model with immigration under conditions of below replacement fertility $[4,7,19]$, on the other hand on the theory of age-structured epidemic models $[2,6,9,13]$.

We will therefore assume that the population demographic rates are below replacement (in the demographic literature the acronym BRF, below replacement fertility is often used), but that an immigration inflow helps in in driving the population to stationarity. In order to focus the attention on the interaction between epidemiologgy and demography per se, and not on the interaction among social groups, we assume that immigrants are indistinguishable by residents, as they arrive. The current setting can be easily extended to more realistic assumptions, such as considering separately residents and immigrants, but letting the children of the latter (first-generation) be identical to residents, but the resulting models would certainly be more complex. Finally, we assume, following the usual approach in models for infectious diseases aiming at analytical results [8, 9, 13], that the population is in a demographically stationary state. Hence, we assume that the population has reached the equilibrium $[4,7,12]$ between below-replacement-fertility and immigration. This assumption allows us to obtain the results discussed in Sections 4 and 5;

Supported in part by FIRB project RBAU01K7M2 "Metodi dell'Analisi Matematica in Biologia, Medicina e Ambiente" of the Italian Ministero Istruzione Università e Ricerca. 
in Section 6, we present the results of some simulations that compare the epidemic processes obtained under the assumption of a demographically stationary state, with those that start from an initial state close to the current Italian demography and that have a very long transient period.

Threshold theorems are an important contribution of mathematical epidemiology, starting from the pioneering work of Ross and Kermack-McKendrick. For the SIR model, without age structure, one can refer to Hethcote [10]. The case of the age-structured SIR model has been thoroughly analysed by Inaba [13] in terms of $R_{0}$, defined as the spectral radius of a certain positive linear operator: when $R_{0} \leq 1$, there exists only the disease-free equilibrium (DFE), and it is globally stable; on the other hand, when $R_{0}>1$, the DFE is unstable, and there exists (at least one) endemic equilibrium.

How do the properties of the model change when immigration is included in the model? It is rather clear that, if a constant flow of infectives enters the population, there cannot be a disease-free equilibrium. Brauer and Van den Driessche $(2001,[5])$ in their analysis of an SIR model, without age structure, but with a constant flow of new members into the population a fraction $p$ of which is infective, started from the observation that, if $p>0$ there is a unique endemic equilibrium $u^{*}(p)$ for all parameter values. However, they recovered a threshold-like behaviour in the following sense: as $p$ goes to zero, if $\mathcal{R}_{0}<1$, then $u^{*}(p)$ tends to the DFE as $p$ goes to zero; otherwise if $\mathcal{R}_{0}>1$ then for $p=0$ the model has a unique endemic equilibrium $u^{*}$, and $u^{*}(p)$ tends to $u^{*}$ as $p$ goes to zero. $R_{0}$ is defined as in the SIR model without immigration [10].

In this paper we extend the threshold result by Inaba [13] considering immigration of infective individuals, in the same spirit as Brauer and Van den Driessche [5]. We then briefly discuss conditions that guarantee the uniqueness of the steady state in this model. The equilibrium dynamics of age-structured SIR models in the framework of populations with BRF and immigration has already been considered in Iannelli and Manfredi [11] but under the assumption of proportionate mixing. In this paper we consider instead fully general age structured mixing patterns that include what is generally used in realistic simulations of childhood diseases (see, for instance, [3]).

\section{The EQUations of The MODEL.}

We start from the equations for a population with age-strucured fertility and mortality rates $m(a), \mu(a)$ and subject to a constant (as age structure and total number of individuals) immigration inflow $I(a)$. The evolution of the density $n(a, t)$ of individuals aged $a$ at time $t$ can be described by the following McKendrick-Von Foerster PDE with boundary and initial conditions:

$$
\left\{\begin{aligned}
\left(\frac{\partial}{\partial t}+\frac{\partial}{\partial a}\right) n(a, t) & =-\mu(a) n(a, t)+I(a) \quad 0<a<\omega, t>0 \\
n(0, t) & =B(t)=\int_{0}^{\omega} m(a) n(a, t) d a \quad t \geq 0 \\
n(a, 0) & =n_{0}(a) \quad 0<a<\omega
\end{aligned}\right.
$$

In (1) $\omega$ represents the maximum life span of the individuals, and $B(t)$ is the number of newborns per unit time at time t. Technical conditions to ensure the well-posedness of the problem are that the mortality rate $\mu(a)$ lies in $L_{l o c}^{1}([0, \omega))$, with $\int_{0}^{\omega} \mu(a) d a=+\infty$, and that the fertility rate $m(\cdot) \in L^{\infty}(0, \omega)$.

We will consider these equations under the conditions of below replacement fertility (BRF). If $\Pi(a)=e^{-\int_{0}^{a} \mu(s) d s}$ is the proportion of individuals who are still living at age a (because of the assumptions on $\mu(\cdot)$, we have $\Pi(\omega)=0$ ), $R=\int_{0}^{\omega} m(a) \Pi(a) d a$ represents the net reproduction rate, i.e. the average number 
of newborn individuals an individual is expected to produce during his reproductive life. The BRF condition is then $R<1$. Equation (1) is the basis of the so called stable population model with immigration (SPI model), used in demography to analyze the long term behaviour of populations with BRF and subject to immigration [4]. Cerone [7] and Inaba [12] proved that the stationary solution of (1), given by

$$
n(a)=B \Pi(a)+\int_{0}^{\omega} I(s) \frac{\Pi(a)}{\Pi(s)} d s, \quad 0 \leq a \leq \omega
$$

with $B$ given by:

$$
B=\frac{1}{1-R} \int_{0}^{\omega} m(a) \Pi(a) \int_{0}^{a} \frac{I(s)}{\Pi(s)} d s d a,
$$

is globally asymptotically stable.

As stated in the introduction, we study the spread of an epidemic under the condition that the population is in the demographical stationary state $(2)$. We consider a SIR age-structured epidemic model where $X(a, t), Y(a, t), Z(a, t)$ are the densities of susceptible, infective and removed individuals of age a at time $t$; we assume that the contact process between individivduals is summarised by the transmission coefficient $\beta\left(a, a^{\prime}\right)$, that is $\beta\left(a, a^{\prime}\right) X(a, t) Y\left(a^{\prime}, t\right) d a d a^{\prime}$ is the number of susceptibles aged in $(a, a+d a)$ that contract the disease by means of a suitable contact with an infective aged in $\left(a^{\prime}, a^{\prime}+d a^{\prime}\right)$ in the time unit at time t: i.e. we assume for the force of infection $\lambda(a, t)$ (FOI for short) the functional form (see $[2,24])$

$$
\lambda(a, t)=\int_{0}^{\omega} \beta(a, s) Y(s, t) d s .
$$

Remark 1. The functional form (4) basically assumes that the force of infection scales linearly with population density. As observed by Manfredi and Williams [20], such a choice makes the force of infection too much sensitive with respect to changes in population size, in case of demographical instabilities. Other choices have been used in the literature, such as

$$
\lambda(a, t)=\int_{0}^{\omega} \beta(a, s) \frac{Y(s, t)}{n(s, t)} d s \quad \text { or } \quad \lambda(a, t)=\frac{1}{N(t)} \int_{0}^{\omega} \beta(a, s) Y(s, t) d s .
$$

If population density is stationary, different choices correspond to a redifinition of $\beta$, and we may in any case study the problem using (4). If, on the other hand, population density fluctuates, different choices for $\lambda(a, t)$ may give rise to different qualitative behaviours; this will be explored in the future through simulations.

We denote $I_{X}(a), I_{Y}(a), I_{Z}(a)$ the densities of susceptible, infective and removed immigrants that enter the population in the time unit. For consistency with equation (1), we require $I_{X}(a)+I_{Y}(a)+I_{Z}(a)=I(a)$.

Finally, we let $\gamma$ is the removal rate, so that $1 / \gamma$ is the average infectious period. Then the spread of the disease can be described by the following non homogeneous system of PDE's a-la Lotka-McKendrick:

(6a) $\left\{\begin{aligned}\left(\frac{\partial}{\partial t}+\frac{\partial}{\partial a}\right) X & =-(\lambda(a, t)+\mu(a)) X+I_{X}(a) \\ \left(\frac{\partial}{\partial t}+\frac{\partial}{\partial a}\right) Y & =\lambda(a, t) X-(\mu(a)+\gamma) Y+I_{Y}(a) \quad 0<a<\omega, t>0 \\ \left(\frac{\partial}{\partial t}+\frac{\partial}{\partial a}\right) Z & =\gamma Y-\mu(a) Z+I_{Z}(a)\end{aligned}\right.$

To complete system (6), boundary and initial conditions are to be given

$$
X(0, t)=B, Y(0, t)=0, Z(0, t)=0
$$


with $B$ given by (3). Finally, the initial conditions are

(6c) $\quad X(a, 0)=X_{0}(a), Y(a, 0)=Y_{0}(a), Z(a, 0)=Z_{0}(a)$

$0<a<\omega$.

Condition (6b) amounts to assuming that all newborn individuals are susceptibles, that is we assume there is no vertical transmission of the disease (nor, maternally transmitted immunity).

Moreover, the initial densities (6a) satisfy $X_{0}(a)+Y_{0}(a)+Z_{0}(a)=n(a)$, the stationary density. Hence, we have

$$
X(a, t)+Y(a, t)+Z(a, t)=n(a) \forall t \geq 0 .
$$

The problem to be considered is then given by (6), completed by the relation (4). Well-posedness can be obtained, as in [13] by setting it as an initial value problem in the Banach space $L^{1}(0, \omega)$. Using standard methods, one can obtain

Theorem 1. Let $I_{x}, I_{y}, I_{z} \in L_{+}^{1}(0, \omega), \beta \in L^{\infty}((0, \omega) \times(0, \omega)) \geq 0$. Assume that the initial data $X_{0}, Y_{0}$ satisfy

$$
\begin{array}{r}
X_{0}(\cdot), Y_{0}(\cdot) \in A C([0, \omega]) \text { with } X_{0}(0)=B, Y_{0}(0)=0, \\
X_{0}(a) \geq 0, Y_{0}(a) \geq 0 \text { a.e. } a \in(0, \omega) .
\end{array}
$$

Then there exists one and only one classical solution of system (6)-(4), which is defined for all $t \geq 0$.

\section{Steady states.}

We now consider existence, uniqueness and threshold behaviour of steady states of system (6). We start by making suitable assumptions on the contact coefficient $\beta(\cdot, \cdot)$ and the age profile of the immigrants to be used.

We assume again $I_{x}, I_{y}, I_{z} \in L^{1}(0, \omega), \beta \in L^{\infty}((0, \omega) \times(0, \omega))$ to be nonnegative functions. Further we assume

Assumption 1. $\beta(\cdot, \cdot)$ satisfies

$$
\lim _{h \rightarrow 0} \int_{0}^{\omega}|\beta(a+h, s)-\beta(a, s)| d a=0
$$

uniformly for $s \in \mathrm{R}$, with $\beta(\cdot, \cdot)$ extended by setting $\beta(a, s)=0$ for $a, s \in(-\infty, 0) \cup$ $(\omega,+\infty)$

Assumption 2. There exists $m>0,0<\alpha<\omega$ such that $\beta(a, s) \geq m$ for a.e. $(a, s) \in(0, \omega) \times(\omega-\alpha, \omega)$.

Assumption 3. There exist $0 \leq a_{1}<a_{2} \leq \omega$ such that $I_{y}(a)>0$ a.e. $a \in\left(a_{1}, a_{2}\right)$.

The equations for the steady states of (6) are as follows:

$$
\left\{\begin{array}{l}
\frac{d}{d a} X^{*}(a)=-\left(\lambda^{*}(a)+\mu(a)\right) X^{*}(a)+I_{x}(a) \\
\frac{d}{d a} Y^{*}(a)=\lambda^{*}(a) X^{*}(a)-(\gamma+\mu(a)) Y^{*}(a)+I_{y}(a)
\end{array}\right.
$$

with

$$
\lambda^{*}(a)=\int_{0}^{\omega} \beta(a, s) Y^{*}(s) d s .
$$


By solving (8) directly we obtain:

$$
\begin{aligned}
& X^{*}(a)=B \pi(a) e^{-\int_{0}^{a} \lambda^{*}(s) d s}+\int_{0}^{a} I_{x}(\sigma) \frac{\pi(a)}{\pi(\sigma)} e^{-\int_{\sigma}^{a} \lambda^{*}(s) d s} d \sigma \\
& Y^{*}(a)=\int_{0}^{a} e^{-\gamma(a-\sigma)} \frac{\pi(a)}{\pi(\sigma)}\left(\lambda^{*}(\sigma) X^{*}(\sigma)+I_{y}(\sigma)\right) d \sigma
\end{aligned}
$$

Then we obtain for the force of infection $\lambda^{*}(\cdot)$ :

$$
\begin{aligned}
\lambda^{*}(a) & =\int_{0}^{\omega} \beta(a, \xi) Y^{*}(\xi) d \xi=\int_{0}^{\omega}\left(B \lambda^{*}(\sigma) e^{-\int_{0}^{\sigma} \lambda^{*}(s) d s} \pi(\sigma)\right. \\
& \left.+\lambda^{*}(\sigma) \int_{0}^{\sigma} I_{x}(s) e^{-\int_{s}^{\sigma} \lambda^{*}(\tau) d \tau} \frac{\pi(\sigma)}{\pi(s)} d s+I_{y}(\sigma)\right) \phi(a, \sigma) d \sigma
\end{aligned}
$$

where $\phi(\cdot, \cdot)$ is given by:

$$
\phi(a, \sigma)=\int_{\sigma}^{\omega} \beta(a, \xi) e^{-\gamma(\xi-\sigma)} \frac{\pi(\xi)}{\pi(\sigma)} d \xi .
$$

We consider

$$
L_{+}^{1}(0, \omega)=\left\{f \in L^{1}(0, \omega) \text { s.t. } f \geq 0 \text { a.e. on }(0, \omega)\right\},
$$

the cone of the nonnegative functions in the Banach space $L^{1}(0, \omega)$, and define the positive nonlinear operator $\Phi: L_{+}^{1}(0, \omega) \longrightarrow L_{+}^{1}(0, \omega)$ by setting

$$
\begin{aligned}
(\Phi \psi)(a) & =\int_{0}^{\omega}\left(B \psi(\sigma) \pi(\sigma) e^{-\int_{0}^{\sigma} \psi(s) d s}\right. \\
& \left.+\psi(\sigma) \int_{0}^{\sigma} I_{x}(s) e^{-\int_{s}^{\sigma} \psi(\tau) d \tau} \frac{\pi(\sigma)}{\pi(s)} d s+I_{y}(\sigma)\right) \phi(a, \sigma) d \sigma
\end{aligned}
$$

$a \in(0, \omega)$.

Each fixed point of $\Phi$ in the positive cone is a force of infection $\lambda^{*}(\cdot)$ that satisfies (10); hence, through (9), it yields an equilibrium solution of (6); and viceversa.

Just by looking at $(9 \mathrm{~b})$ we see that, because of assumption 3 , in any equilibrium solution of $(6)$ the density infectives $Y^{*}(\cdot)$ satisfies $Y^{*}>0$. This means that in presence of infectives in the immigrant population, there is no disease-free equilibium (DFE), as was noted by Brauer-Van den Driessche [5] for a SIR model without age structure.

Furthermore, if we define

$$
u_{0}(a)=(\Phi(0))(a)=\int_{0}^{\omega} I_{y}(\sigma) \phi(a, \sigma) d \sigma, a \in(0, \omega)
$$

we see that any fixed point $\lambda^{*}$ of (13) satisfies $\lambda^{*}(a) \geq u_{0}(a)$ a.e. Under Assumption 2 , we have

(15) $\phi(a, \sigma) \geq m \int_{\max \{\sigma, \omega-\alpha\}}^{\omega} e^{-\gamma(\xi-\sigma)} \frac{\pi(\xi)}{\pi(\sigma)} d \xi \geq m e^{-\gamma \omega} \int_{\max \{\sigma, \omega-\alpha\}}^{\omega} \pi(\xi) d \xi=\phi_{m}(\sigma)$.

It is easy to see that $\phi_{m}(\sigma)>0$ for all $\sigma<\omega$; moreover, $\phi_{m}(\cdot)$ is continuous and non-increasing. Then, because of Assumption 3,

$$
u_{0}(a) \geq \int_{0}^{\omega} I_{y}(\sigma) \phi_{m}(\sigma) d \sigma=\underline{u}>0 .
$$

This means that at an equilibrium solution of (6), the force of infection $\lambda^{*}$ is strictly positive at all ages.

We study fixed points of (13) using the theory of positive operators defined on a cone in a Banach space.

We first note that there exists $R>0$ such that $\|\Phi \psi\|_{1} \leq R \forall \psi \in L_{+}^{1}(0, \omega)$. 
In fact, using the definition, we have

$$
\phi(a, \sigma) \leq\|\phi\|_{\infty} \leq\|\beta\|_{\infty} \omega .
$$

Then,

$$
\begin{aligned}
& \Phi(\psi)(a) \leq\|\phi\|_{\infty}\left(B \int_{0}^{\omega} \psi(\sigma) e^{-\int_{0}^{\sigma} \psi(s) d s} d \sigma\right. \\
&\left.+\int_{0}^{\omega} I_{x}(s) \int_{s}^{\omega} \psi(\sigma) e^{-\int_{s}^{\sigma} \psi(\tau) d \tau} d \sigma d s+\left\|I_{y}\right\|_{1}\right) \\
&=\|\phi\|_{\infty}\left(B\left(1-e^{-\int_{0}^{\omega} \psi}\right)+\int_{0}^{\omega} I_{x}(s)\left(1-e^{-\int_{s}^{\omega} \psi} d s\right)+\left\|I_{y}\right\|_{1}\right) \\
& \leq\|\phi\|_{\infty}\left(B+\left\|I_{x}\right\|_{1}+\left\|I_{y}\right\|_{1}\right),
\end{aligned}
$$

and $R$ can be obtained easily.

Define now

$$
D=\left\{\psi \in L_{+}^{1}(0, \omega):\|\psi\|_{1} \leq R\right\} \cap\left\{\psi \in L_{+}^{1}(0, \omega): \psi \geq u_{0}\right\} .
$$

We have the following

Theorem 2. Let assumptions 1-3 hold. Then

i): $D$ is closed, bounded, convex and such that $\Phi(D) \subseteq D$;

ii): $\Phi$ is completely continuous.

Hence, there exists $\psi \in D$ such that $\psi=\Phi(\psi)$.

Proof. i) follows immediately from the definition, and the previous considerations. ii) There exists $C>0$ s.t. $\left\|\Phi\left(\psi_{1}\right)-\Phi\left(\psi_{2}\right)\right\|_{1} \leq C\left\|\psi_{1}-\psi_{2}\right\|_{1} \forall \psi_{1}, \psi_{2} \in L_{+}^{1}(0, \omega)$; in fact

$$
\begin{aligned}
& \left(\Phi\left(\psi_{1}\right)\right)(a)-\left(\Phi\left(\psi_{2}\right)\right)(a) \\
& =\int_{0}^{\omega} B\left(\psi_{1}(\sigma) e^{-\int_{0}^{\sigma} \psi_{1}(\tau) d \tau}-\psi_{2}(\sigma) e^{-\int_{0}^{\sigma} \psi_{2}(\tau) d \tau}\right) \pi(\sigma) \phi(a, \sigma) d \sigma \\
& \quad+\int_{0}^{\omega} I_{x}(s) \int_{s}^{\omega} \frac{\pi(\sigma)}{\pi(s)}\left(\psi_{1}(\sigma) e^{-\int_{s}^{\sigma} \psi_{1}}-\psi_{2}(\sigma) e^{-\int_{s}^{\sigma} \psi_{2}}\right) \phi(a, \sigma) d \sigma d s .
\end{aligned}
$$

For the first term in (18) we have

$$
\begin{aligned}
& \left|B \int_{0}^{\omega} \pi(\sigma)\left(\psi_{1}(\sigma) e^{-\int_{0}^{\sigma} \psi_{1}}-\psi_{2}(\sigma) e^{-\int_{0}^{\sigma} \psi_{2}}\right) \phi(a, \sigma) d \sigma\right| \\
& \quad \leq\|\phi\|_{\infty} B\left|\int_{0}^{\omega} \frac{d}{d \sigma}\left(e^{-\int_{0}^{\sigma} \psi_{2}}-e^{-\int_{0}^{\sigma} \psi_{1}}\right) d \sigma\right| \\
& \quad=\|\phi\|_{\infty}\left|e^{-\left\|\psi_{2}\right\|_{1}}-e^{-\left\|\psi_{1}\right\|_{1}}\right| \leq\|\phi\|_{\infty}\left\|\psi_{2}-\psi_{1}\right\|_{1} \quad \text { a.e. } a \in(0, \omega)
\end{aligned}
$$

and a similar inequality holds for the second term in (18). Then in particular $\Phi$ is continuous.

Let us consider the operator $T: L^{1}(0, \omega) \longrightarrow L^{1}(0, \omega)$ defined as

$$
(T \psi)(a)=\int_{0}^{\omega} \psi(\sigma)\left(B \pi(s)+\int_{0}^{\sigma} I_{x}(s) \frac{\pi(\sigma)}{\pi(s)} d s\right) \phi(a, \sigma) d \sigma
$$

for $a \in(0, \omega), \psi \in L^{1}(0, \omega)$.

$T$ is linear, continuous and positive (furthermore, $T$ is the Frechet derivative of $\Phi$ in zero, $\left.\Phi^{\prime}(0)\right)$. From Assumption 1 and the Riesz-Frechet-Kolmogorov theorem on compactness, it follows that $T$ is a compact operator.

Compactness of $\Phi$ now follows from the inequality

$$
u_{0} \leq \Phi \psi \leq T \psi+u_{0} \quad \forall \psi \in L_{+}^{1}(0, \omega) .
$$


Hence $\Phi$ is completely continuous.

From (i) we know that $\Phi(D) \subseteq D$, with $D$ closed, bounded and convex. From Schauder's principle it follows that $\Phi$ has at least a fixed point in $D$.

\section{Threshold-Like Results.}

In the SIR model without immigration Inaba [13] proved that the spectral radius of $T$ is a threshold for the infection. He proved

Theorem A (Inaba).

1) If $\rho(T) \leq 1$, the only fixed point of the operator $\Phi$ is the null vector $\psi \equiv 0$;

2) if $\rho(T)>1$ there is at least a non-zero fixed point of $\Phi$.

If the rate of infected immigrants $I_{y}$ is not zero, then we know (Theor. 2) that a positive equilibrium is present both if $\rho(T)>1$ and if $\rho(T) \leq 1$, and that there exists no DFE.

In the SIR model without immigration it is still possible to distinguish between two different situations for the steady states in terms of the limiting behaviour of the fixed points of $\Phi$ as $\left\|u_{0}\right\|_{1}$ goes to zero.

We proceed as follows: for fixed $I_{x}(\cdot), I_{z}(\cdot)$, we consider a sequence $I_{y, n} \in$ $L_{+}^{1}(0, \omega)$ such that $I_{y, n}(a) \underset{n}{\longrightarrow} 0$ a.e. $a \in(0, \omega)$.

$\Phi_{n}$ and $u_{n}$ are the analogous of (13), (14) in which $I_{y, n}$ has been inserted in place of $I_{y}$ with the corresponding change in the stationary population $B_{n}$ because of (3). Moreover, we let $\Phi_{0}$ to be defined as (13) with $I_{y}=0$.

We also define $\tilde{\Phi}_{n}=\Phi_{n}-u_{n}$, noting that $\tilde{\Phi}_{n}$ depends on $n$ only because of the term $B_{n}$. Finally, consider the positive linear operators on $L^{1}(0, \omega) T_{n}=\Phi_{n}^{\prime}(0)$, $T_{0}=\Phi_{0}^{\prime}(0):$

$$
\begin{aligned}
& \left(T_{n} \psi\right)(a)=\int_{0}^{\omega} \psi(\sigma)\left(B_{n} \pi(\sigma)+\int_{0}^{\sigma} I_{x}(s) \frac{\pi(\sigma)}{\pi(s)} d s\right) \phi(a, \sigma) d \sigma \\
& \left(T_{0} \psi\right)(a)=\int_{0}^{\omega} \psi(\sigma)\left(B_{0} \pi(\sigma)+\int_{0}^{\sigma} I_{x}(s) \frac{\pi(\sigma)}{\pi(s)} d s\right) \phi(a, \sigma) d \sigma
\end{aligned}
$$

Consider a sequence $\left\{\psi_{n}\right\} \subset L_{+}^{1}(0, \omega)$, where $\psi_{n}$ is a fixed point for $\Phi_{n}$. Our main result is the following

\section{Theorem 3.}

1) If $\rho\left(T_{0}\right) \leq 1$ then $\lim _{n \rightarrow \infty} \psi_{n}=0$;

2) if $\rho\left(T_{0}\right)>1$ then there exists $\delta>0$ such that $\left\|\psi_{n}\right\|_{1} \geq \delta \forall n \in \mathbb{N}$.

Before proving the theorem, we need some preliminaries. First, we use the following

Proposition 4. There exists a converging subsequence of $\left\{\psi_{n}\right\}$. Let $\psi=\lim _{k \rightarrow \infty} \psi_{n_{k}}$ for any converging subsequence $\left\{\psi_{n_{k}}\right\}$; then $\psi=\Phi_{0}(\psi)$. In other words, the set of limit points of $\left\{\psi_{n}\right\}$ are fixed points for $\Phi_{0}$.

Proof. Since $0 \leq\left\|\psi_{n}\right\| \leq R$, where $R=\max _{n} R_{n}$ found from (16), and $\Phi_{0}$ is compact, there exists a converging subsequence $\left\{\Phi_{0}\left(\psi_{n_{k}}\right)\right\}$.

Let $\psi=\lim _{k \rightarrow \infty} \Phi_{0}\left(\psi_{n_{k}}\right)$. We have

$$
\psi_{n_{k}}=\Phi_{n_{k}}\left(\psi_{n_{k}}\right)=\Phi_{0}\left(\psi_{n_{k}}\right)+\left(\Phi_{n_{k}}\left(\psi_{n_{k}}\right)-\Phi_{0}\left(\psi_{n_{k}}\right)\right) .
$$


Furthermore

$$
\begin{aligned}
& \left(\Phi_{n_{k}}\left(\psi_{n_{k}}\right)\right)(a)-\left(\Phi_{0}\left(\psi_{n_{k}}\right)\right)(a) \\
& =\left(B_{n_{k}}-B_{0}\right) \int_{0}^{\omega} \psi_{n_{k}}(\sigma) \pi(\sigma) e^{-\int_{0}^{\sigma} \psi_{n_{k}}(\tau) d \tau} \phi(a, \sigma) d \sigma \\
& \quad+\int_{0}^{\omega} I_{y, n_{k}}(\sigma) \phi(a, \sigma) d \sigma .
\end{aligned}
$$

Since, by assumption, $\lim _{n \rightarrow \infty} I_{y, n}=0$ a.e., and clearly from (3) $\lim _{n \rightarrow \infty} B_{n}=B_{0}$, $\lim _{k \rightarrow \infty}\left[\Phi_{n_{k}}\left(\psi_{n_{k}}\right)-\Phi_{0}\left(\psi_{n_{k}}\right)\right]=0$ a.e., hence in $L^{1}$.

Substituting this in (22), we have

$$
\lim _{k \rightarrow \infty} \psi_{n_{k}}=\lim _{k \rightarrow \infty} \Phi_{0}\left(\psi_{n_{k}}\right)=\psi
$$

so that $\left\{\psi_{n_{k}}\right\}$ converges to $\psi$. Furthermore, since $\Phi_{0}$ is continuous, $\lim _{k \rightarrow \infty} \Phi_{0}\left(\psi_{n_{k}}\right)=\Phi_{0}(\psi)$ and $\psi$ is a fixed point for $\Phi_{0}$.

The same arguments can be applied to any converging subsequence $\left\{\psi_{n_{k}}\right\}$.

We also need some results about the spectral theory of positive operators, that we briefly recall.

Definition 1. Let $E$ be a Banach space, $K \subset E$ a cone. The cone $K$ is called total if it satisfies

$$
\overline{K-K}=\overline{\{\psi-\varphi: \psi, \varphi \in K\}}=E .
$$

Theorem B (Krein-Rutman (1948)). Let E be a real Banach space with the total order cone $K$. Let $A: E \longrightarrow E$ be linear, compact and positive with respect to $K$, and with $\rho(A)>0$. Then $\rho(A)$ is an eigenvalue of $A$ and $A^{*}$ with eigenvectors in $K, K^{*}$, respectively.

Krein and Rutman [17] present several conditions that ensure the positivity of $\rho(A)$, and guarantee that it has multiplicity 1 . Since $L_{+}^{1}(0, \omega)$ has empty interior, we cannot use their results, but instead use the following theorem on kernel operators.

$$
A f(t)=\int K(s, t) f(s) d \mu(s)
$$

Theorem C ([23], Theor.V6.6). Let $E=L^{p}(\mu)$, where $1 \leq p \leq+\infty$ and $(X, \Sigma, \mu)$ is a $\sigma$-finite measure space. Suppose $A \in \mathcal{L}(E)$ has the form (23) where $K \geq 0$ is a $\Sigma \times \Sigma$-measurable kernel, satisfying the two assumptions:

i) some power of $A$ is compact;

ii) $S \in \Sigma$ and $\mu(S)>0, \mu(X \backslash S)>0$ implies

$$
\int_{X \backslash S} \int_{S} K(s, t) d \mu(s) d \mu(t)>0 .
$$

Then $\rho(A)>0$ is an eigenvalue of $A$ with a unique normalized eigenfunction $f$ satisfying $f(s)>0 \mu$-a.e.; moreover if $K(s, t)>0(\mu \otimes \mu)$-a.e. then every other eigenvalue $\lambda$ of $A$ has modulus $|\lambda|<\rho(A)$.

We can apply this theorem to $T_{0}$ obtaining

Lemma 5. $\rho\left(T_{0}\right)$ is an eigenvalue of $T_{0}$ and $T_{0}^{*}$ with unique normalized strictly positive eigenfunctions $\psi$ and $f$. 
Proof. $T_{0}$ is a kernel operator and, as shown in the proof of Theorem 2, is compact. Moreover, from (15), it is easy to see that condition (24) is satisfied. Then Theorem $\mathrm{C}$ applies, so that $\rho\left(T_{0}\right)>0$ and $\rho\left(T_{0}\right)$ is the only eigenvalue of $T_{0}$ of maximal modulus with a unique normalized eigenvector $\psi \in L_{+}^{1}(0, \omega)$ and satisfying $\psi(a)>0$ a.e.

$T_{0}^{*}$ has the same non zero eigenvalues as $T_{0}$ with the same multiplicity. Thus $\rho\left(T_{0}\right)$ is an algebraically simple eigenvalue of $T_{0}^{*}$ and by Theorem B $\left(L_{+}^{1}(0, \omega)\right.$ is a total cone) it corresponds to a (unique normalized) eigenfunctional $f$. It remains to be proved that $f$ is strictly positive.

$T_{0}^{*}$ acts from $L^{\infty}(0, \omega)$ in itself as follows:

$$
\left(T_{0}^{*} \varphi\right)(a)=\int_{0}^{\omega} \varphi(\sigma)\left(B_{0} \pi(a)+\int_{0}^{a} I_{x}(s) \frac{\pi(a)}{\pi(s)} d s\right) \phi(\sigma, a) d \sigma .
$$

Using Assumption 2 as in (15), it can be proved that there exists a continuous function $g:[0, \omega] \longrightarrow \mathbb{R}$ s.t. $g(\omega)=0, g(a)>0 \forall a \in[0, \omega)$ such that

$$
\left(B_{0} \pi(a)+\int_{0}^{a} I_{x}(s) \frac{\pi(a)}{\pi(s)} d s\right) \phi(\sigma, a) \geq g(a) \quad \text { a.e. } a, \sigma \in(0, \omega) .
$$

Then

$$
f(a)=\frac{1}{\rho\left(T_{0}\right)}\left(T_{0}^{*} f\right)(a) \geq \frac{1}{\rho\left(T_{0}\right)} g(a) \int_{0}^{\omega} f(\sigma) d \sigma>0 \quad \text { a.e. } a \in(0, \omega)
$$

because $f \in L_{+}^{\infty}(0, \omega) \backslash\{0\}$. Then $f$ is strictly positive.

Remark 2. This fact can be stated in a more general context by observing that, as a consequence of Assumption 2, $T_{0}$ is an irreducible operator on the Banach lattice $L^{1}(0, \omega)$ ([23], V.6). This roughly corresponds to the definition of non-supporting operator used by Inaba [13].

Lemma 6. The following inequality holds:

$$
e^{-\|\psi\|_{1}} T_{0} \psi \leq \tilde{\Phi} \psi \leq T_{0} \psi \quad \forall \psi \in L_{+}^{1}(0, \omega)
$$

Proof. Let $\psi \in L_{+}^{1}(0, \omega)$, then:

$$
\begin{aligned}
(\tilde{\Phi} \psi)(a) & =\int_{0}^{\omega} \psi(\sigma) \pi(\sigma)\left(B_{0} e^{-\int_{0}^{\sigma} \psi(s) d s}+\int_{0}^{\sigma} \frac{I_{x}(s)}{\pi(s)} e^{-\int_{s}^{\sigma} \psi(\tau) d \tau} d s\right) \phi(a, \sigma) d \sigma \\
& \leq \int_{0}^{\omega} \psi(\sigma) \pi(\sigma)\left(B_{0}+\int_{0}^{\sigma} \frac{I_{x}(s)}{\pi(s)} d s\right) \phi(a, \sigma) d \sigma=\left(T_{0} \psi\right)(a) ; \\
(\tilde{\Phi} \psi)(a) & \geq e^{-\int_{0}^{\omega} \psi(s) d s} \int_{0}^{\omega} \psi(\sigma) \pi(\sigma)\left(B_{0}+\int_{0}^{\sigma} \frac{I_{x}(s)}{\pi(s)} d s\right) \phi(a, \sigma) d \sigma \\
& =e^{-\|\psi\|_{1}}\left(T_{0} \psi\right)(a) .
\end{aligned}
$$

Remark 3. By induction a more general inequality can be proved:

$$
e^{-\sum_{k=0}^{n-1}\left\|T_{0}^{k} \psi\right\|_{1}} T_{0}^{n} \psi \leq \tilde{\Phi}^{n} \psi \leq T_{0}^{n} \psi \quad \forall \psi \in L_{+}^{1}(0, \omega), \forall n \in \mathbb{N} .
$$

All of what has been proved for $T_{0}$, holds obviously also for $T_{n}, \forall n \in \mathbb{N}$. Moreover,

Lemma 7. $\lim _{n \rightarrow \infty} \rho\left(T_{n}\right)=\rho\left(T_{0}\right)$, and $\rho\left(T_{n}\right) \geq \rho\left(T_{0}\right)$ for all $n$.

Proof. As seen above $B_{n} \underset{n}{\longrightarrow} B_{0}$, hence $T_{n}$ tends to $T_{0}$ uniformly. Since they are compact positive operators, $\rho\left(T_{n}\right)$ [and $\rho\left(T_{0}\right)$ ] are isolated eigenvalues of $T_{n}$ [and $T_{0}$ ]. Then standard results in perturbation theory (see [15], Section IV.3.5) show that $\rho\left(T_{n}\right) \underset{n}{\longrightarrow} \rho\left(T_{0}\right)$. 
As for the second claim, from (3), one sees $B_{0}<B_{n}$, hence $T_{0} \leq T_{n}$. From a comparison result about positive operators (see [22]) then follows that $\rho\left(T_{n}\right) \geq$ $\rho\left(T_{0}\right)$.

We can now prove the main threshold result:

\section{of Theorem 3.}

i) Using Proposition 4, we see that any converging subsequence $\left\{\psi_{n_{k}}\right\} \subset\left\{\psi_{n}\right\}$ tends to $\psi$ fixed point of $\Phi_{0}$. Since, for $\rho\left(T_{0}\right) \leq 1, \Phi_{0}$ has only 0 as fixed point (Theorem A), $\psi=0$, i.e. the whole sequence converges to 0 .

ii) From $\rho\left(T_{0}\right)>1$ it follows that $\rho\left(T_{n}\right)>1 \forall n$ by virtue of Lemma 7 . Let $f_{n} \in\left(L_{+}^{1}(0, \omega)\right)^{*} \backslash\{0\}$ be the strictly positive eigenvector of $T_{n}^{*}$ with respect to the eigenvalue $\rho\left(T_{n}\right)$. Then we have, $\forall n \in \mathbb{N}$,

$$
\begin{aligned}
<f_{n}, \psi_{n}> & =<f_{n}, \Phi_{n} \psi_{n}>=<f_{n}, \tilde{\Phi}_{n} \psi_{n}+u_{n}> \\
& \geq<f_{n}, e^{-\left\|\psi_{n}\right\|_{1}} T_{n} \psi_{n}+u_{n}>><f_{n}, e^{-\left\|\psi_{n}\right\|_{1}} T_{n} \psi_{n}> \\
& =e^{-\left\|\psi_{n}\right\|_{1}}<T_{n}^{*} f_{n}, \psi_{n}>=e^{-\left\|\psi_{n}\right\|_{1}} \rho\left(T_{n}\right)<f_{n}, \psi_{n}>,
\end{aligned}
$$

where the first inequality follows from Lemma 6 . Since $\psi_{n} \geq u_{n}>0$ and $f_{n}$ is a strictly positive functional, we have that:

$$
1>e^{-\left\|\psi_{n}\right\|_{1}} \rho\left(T_{n}\right) \quad \forall n \in \mathbb{N}
$$

that is

$$
\left\|\psi_{n}\right\|_{1}>\log \left(\rho\left(T_{n}\right)\right) \geq \log \left(\rho\left(T_{0}\right)\right) .
$$

The thesis then holds with $\delta=\log \left(\rho\left(T_{0}\right)\right)$.

\section{The issue of Uniqueness.}

Several sufficient conditions guarantee the uniqueness of positive equilibria for the $S I R$ model without immigration, but there exist no general results. Indeed, there exist examples [Franceschetti, in preparation] with multiple positive equilibria of the model without immigration. Hence, we expect that no general results may exist for this model too, and we consider only the extension to this case of the sufficient conditions found for the model without immigration.

Iannelli and Manfredi [11] deal with the case of a separable kernel, i.e. $\beta(a, s)=$ $\beta_{1}(a) \beta_{2}(s)$, where the search for equilibria reduces to a one-dimensional fixed point problem. We consider here instead the other cases, whose proof [13] is based on the concept of monotonicity for sublinear operators [1], which we briefly review.

Given a Banach space $E$ partially ordered by means of a cone $C$, let $u, w \in E$ be such that $u \leq w$, the order interval of extremes $\mathrm{u}, \mathrm{w}$ is the set $[u, w]=\{v \in E: u \leq$ $v \leq w\}=(u+C) \cap(w-C)$. A nonlinear mapping $A: D(A) \subseteq E \longrightarrow E$ is called increasing if, for all $u, v \in D(A)$ such that $u<v$ we have $A u \leq A v$; is called strictly increasing if the strict inequality sign $A u<A v$ holds; and is called e-increasing if there exists $e \in C \backslash\{0\}$ such that for every $u, v \in D(A)$ with $u<v$ there exists constants $\alpha=\alpha(u, v)>0, \beta=\beta(u, v)>0$ such that $\alpha e \leq A v-A u \leq \beta e$.

A nonlinear mapping $A:[v, w] \longrightarrow E$ is called sublinear with respect to $[v, w]$ if the following holds:

$$
A(v+\tau(u-v))-(v+\tau(A u-v)) \geq 0 \forall u \in[v, w], \forall \tau \in[0,1]
$$

A is called strictly sublinear if it holds the strict inequality sign for every $u \in$ $(v, w]=[v, w] \backslash\{v\}$ and $\tau \in(0,1) ; A$ is called e-sublinear if there exists $e \in C \backslash\{0\}$ 
such that for every $u \in(v, w]$ and every $\tau \in(0,1)$ there exists $\delta=\delta(u, \tau)>0$ such that

$$
A(v+\tau(u-v))-(v+\tau(A u-v)) \geq \delta e .
$$

The following uniqueness result is due to Amann [1].

Theorem D. Let $E$ be an ordered Banach space. Let us set $[v, \infty)=\{u \in E$ : $u \geq v\}$ and suppose $A:[v, \infty) \longrightarrow E$ is e-sublinear and e-increasing and suppose there exists $\gamma>0$ such that $0 \leq A v-v \leq \gamma e$. Then $A$ has at most one fixed point in $(v, \infty)=[v, \infty) \backslash\{v\}$.

We can consider the operator $\Phi$ as operating from the order interval $\left[u_{0}, \infty\right)$ into itself, $\Phi:\left[u_{0}, \infty\right) \longrightarrow\left[u_{0}, \infty\right)$, in view of the fact that $\Phi \psi \geq u_{0} \forall \psi \in L_{+}^{1}(0, \omega)$. We have

Proposition 8. $\Phi$ is e-sublinear on $\left[u_{0}, \infty\right)$ with $e(a) \equiv 1$.

Proof. Given $\psi \in L_{+}^{1}(0, \omega)$ with $\psi>u_{0}, \tau \in(0,1)$ we have:

$$
\begin{aligned}
& \Phi\left(u_{0}+\tau\left(\psi-u_{0}\right)\right)(a)-\left(u_{0}+\tau\left(\Phi \psi-u_{0}\right)\right)(a) \\
& =(1-\tau) \int_{0}^{\omega} u_{0}(\sigma)\left(B \pi(\sigma) e^{-\int_{0}^{\sigma}\left((1-\tau) u_{0}(\eta)+\tau \psi(\eta)\right) d \eta}\right. \\
& \left.\quad+\int_{0}^{\sigma} I_{x}(s) e^{-\int_{s}^{\sigma}\left((1-\tau) u_{0}(\eta)+\tau \psi(\eta)\right)} d s\right) \phi(a, \sigma) d \sigma \\
& \quad+\tau \int_{0}^{\omega} \psi(\sigma)\left(B \pi(\sigma) e^{-\int_{0}^{\sigma} \psi(\eta) d \eta}\left(e^{(1-\tau) \int_{0}^{\sigma}\left(\psi(\eta)-u_{0}(\eta)\right) d \eta}-1\right)\right. \\
& \left.\quad+\int_{0}^{\sigma} I_{x}(s) e^{-\int_{s}^{\sigma} \psi}\left(e^{(1-\tau) \int_{s}^{\sigma}\left(\psi(\eta)-u_{0}(\eta)\right) d \eta}-1\right) d s\right) \phi(a, \sigma) d \sigma
\end{aligned}
$$

Since $\phi(a, s) \geq \phi_{m}(s)>0$ a.e. (see (15)), we obtain

$$
\Phi\left(u_{0}+\tau\left(\psi-u_{0}\right)\right)(a)-\left(u_{0}+\tau\left(\Phi \psi-u_{0}\right)\right)(a) \geq \delta
$$

where $\delta=\delta(\psi, \tau)>0$ is the constant obtained substituting $\phi_{m}(s)$ to $\phi(a, s)$ in (29).

Following Inaba [13], an assumption on the kernel $\phi(\cdot, \cdot)$ that guarantees the monotonicity of the operator $\Phi$ is the following

\section{Assumption 4.}

$$
-\frac{d \phi}{d s}(a, s)=\beta(a, s) n(s)-\gamma \phi(a, s) \geq 0 \quad \text { a.e. }(a, s) \in(0, \omega) \times(0, \omega)
$$

We then have

Proposition 9. Under assumption 4, the operator $\Phi$ is e-increasing with respect to the order interval $\left[u_{0}, \infty\right)$.

The proof is identical to that of Inaba.

Hence, we obtain

Theorem 10. Under the assumptions 1-4 there always exists a unique equilibrium of (6).

Proof. The existence part comes from Theorem 2.

As for uniqueness, we use Theorem D. Sublinearity and monotonicity have already been discussed. It remains to check the condition

$$
\exists \gamma>0: 0 \leq \Phi u_{0}-u_{0} \leq \gamma e .
$$

We have $\Phi u_{0}-u_{0}=\tilde{\Phi} u_{0}>0$. Moreover, we have $\left(\Phi u_{0}\right)(a)-u_{0}(a) \leq \gamma e(a)$ a.e. with $e(a) \equiv 1$ as in Proposition 8 and $\gamma=\left\|\Phi u_{0}\right\|_{\infty}+\left\|u_{0}\right\|_{\infty}$. 
Note that, for the case without immigration, Inaba showed, under assumption 4 , that there exists a unique positive equilibrium, if $\rho(T)>1$, and no positive equilibrium if $\rho(T) \leq 1$; moreover, for all parameter values there exists the diseasefree equilibrium. The present results shows clearly the effect of immigration.

Another result of Inaba concerning uniqueness is that, when $\rho(T) \leq 1$, there are no positive equilibria of the system without immigration. A related result holds also for the system with immigration, but requires some qualifications and preliminaries. First, we state the following

Lemma 11. If $\rho(T)<1, \psi \in L_{+}^{1}(0, \omega)$ is a fixed point of $\Phi$ then we have:

$$
(0 \leq) \psi \leq(I-T)^{-1} u_{0} \text {. }
$$

Proof. We have $0 \leq T \psi+u_{0}-\Phi \psi=T \psi+u_{0}-\psi$.

Since $\rho(T)<1$, there exists $(I-T)^{-1}$ and, because $T \in \mathcal{L}_{+}\left(L^{1}(0, \omega)\right),(I-T)^{-1} \in$ $\mathcal{L}_{+}\left(L^{1}(0, \omega)\right)$. Then we have:

$$
0 \leq(I-T)^{-1}\left(T \psi+u_{0}-\psi\right)=(I-T)^{-1}\left(u_{0}-(I-T) \psi\right)=(I-T)^{-1} u_{0}-\psi,
$$

which is the thesis.

The following lemma establishes that, if $\left\|v_{0}\right\|$ is small enough, $\Phi$ is monotone on the order interval $\left[0, v_{0}\right]$.

Proposition 12. Let

$$
\left\|v_{0}\right\| e^{\left\|v_{0}\right\|}<e^{-\gamma \omega} .
$$

Then the operator $\Phi$ is e-increasing on the order interval $\left[0, v_{0}\right]$.

Proof. Given $\psi_{1}, \psi_{2} \in L_{+}^{1}(0, \omega)$ such that $\psi_{1}<\psi_{2} \leq v_{0}$ we have:

$$
\begin{aligned}
& \left(\Phi \psi_{2}\right)(a)-\left(\Phi \psi_{1}\right)(a) \\
& =B \int_{0}^{\omega} \pi(\sigma)\left(\psi_{2}(\sigma)-\psi_{1}(\sigma)\right) e^{-\int_{0}^{\sigma} \psi_{2}(\tau) d \tau} \phi(a, \sigma) d \sigma- \\
& -B \int_{0}^{\omega} \psi_{1}(\sigma) \pi(\sigma)\left(e^{-\int_{0}^{\sigma} \psi_{1}(\tau) d \tau}-e^{-\int_{0}^{\sigma} \psi_{2}(\tau) d \tau}\right) \phi(a, \sigma) d \sigma \\
& +\int_{0}^{\omega} \frac{I_{x}(s)}{\pi(s)} \int_{s}^{\omega}\left(\psi_{2}(\sigma)-\psi_{1}(\sigma)\right) e^{-\int_{s}^{\sigma} \psi_{2}(\tau) d \tau} \phi(a, \sigma) d \sigma d s \\
& -\int_{0}^{\omega} \frac{I_{x}(s)}{\pi(s)} \int_{s}^{\omega} \psi_{1}(\sigma)\left(e^{-\int_{s}^{\sigma} \psi_{1}(\tau) d \tau}-e^{-\int_{s}^{\sigma} \psi_{2}(\tau) d \tau}\right) \phi(a, \sigma) d \sigma d s \\
& \geq B \int_{0}^{\omega} \pi(\sigma)\left(\psi_{2}(\sigma)-\psi_{1}(\sigma)\right) e^{-\int_{0}^{\sigma} \psi_{2}(\tau) d \tau} \phi(a, \sigma) d \sigma \\
& -B \int_{0}^{\omega} \psi_{1}(\sigma) \pi(\sigma) \int_{0}^{\sigma}\left(\psi_{2}(\tau)-\psi_{1}(\tau)\right) d \tau \phi(a, \sigma) d \sigma \\
& +\int_{0}^{\omega} \frac{I_{x}(s)}{\pi(s)} \int_{s}^{\omega} \pi(\sigma)\left(\psi_{2}(\sigma)-\psi_{1}(\sigma)\right) e^{-\int_{s}^{\sigma} \psi_{2}(\tau) d \tau} \phi(a, \sigma) d \sigma d s \\
& -\int_{0}^{\omega} \frac{I_{x}(s)}{\pi(s)} \int_{s}^{\omega} \psi_{1}(\sigma) \pi(\sigma) \int_{s}^{\sigma}\left(\psi_{2}(\tau)-\psi_{1}(\tau)\right) d \tau \phi(a, \sigma) d \sigma d s \\
& =\int_{0}^{\omega}\left(B+\int_{0}^{\sigma} \frac{I_{x}(s)}{\pi(s)} d s\right)\left(\psi_{2}(\sigma)-\psi_{1}(\sigma)\right) \\
& \times\left(\pi(\sigma) e^{-\int_{0}^{\sigma} \psi_{2}\left(\tau^{\prime}\right) d \tau^{\prime}} \phi(a, \sigma)-\int_{\sigma}^{\omega} \psi_{1}(\tau) \pi(\tau) \phi(a, \tau) d \tau\right) d \sigma
\end{aligned}
$$

Moreover, we have, for $\tau \in[\sigma, \omega]$,

$$
\pi(\tau) \phi(a, \tau)=\int_{\tau}^{\omega} \beta(a, \xi) e^{-\gamma(\xi-\tau)} \pi(\xi) d \xi \leq \int_{\sigma}^{\omega} \beta(a, \xi) \pi(\xi) d \xi
$$


Then we obtain

$$
\begin{aligned}
\int_{\sigma}^{\omega} \psi_{1}(\tau) \pi(\tau) \phi(a, \tau) d \tau & \leq\left\|\psi_{1}\right\|_{1} \max _{\tau \in[\sigma, \omega]} \pi(\tau) \phi(a, \tau) \\
& \leq\left\|\psi_{1}\right\|_{1} \int_{\sigma}^{\omega} \beta(a, \xi) \pi(\xi) d \xi
\end{aligned}
$$

and

$$
\begin{aligned}
\pi(\sigma) e^{-\int_{0}^{\sigma} \psi_{2}\left(\tau^{\prime}\right) d \tau^{\prime}} \phi(a, \sigma) & \geq e^{-\left\|\psi_{2}\right\|_{1}} \int_{\sigma}^{\omega} \beta(a, \xi) e^{-\gamma(\xi-\sigma)} \pi(\xi) d \xi \\
& \geq e^{-\left\|\psi_{2}\right\|_{1}} e^{-\gamma \omega} \int_{\sigma}^{\omega} \beta(a, \xi) \pi(\xi) d \xi
\end{aligned}
$$

Substituting (34) and (35) in (33), we obtain

$$
\begin{aligned}
& \left(\Phi \psi_{2}\right)(a)-\left(\Phi \psi_{1}\right)(a) \\
& \quad \geq B\left(e^{-\left\|v_{0}\right\|_{1}} e^{-\gamma \omega}-\left\|v_{0}\right\|_{1}\right) \int_{0}^{\omega}\left(\psi_{2}(\sigma)-\psi_{1}(\sigma)\right) \int_{\sigma}^{\omega} \beta(a, \xi) \pi(\xi) d \xi d \sigma .
\end{aligned}
$$

We have $\psi_{2}>\psi_{1},(32)$ holds, and, because of assumption 2 ,

$$
\int_{\sigma}^{\omega} \beta(a, \xi) \pi(\xi) d \xi \geq \phi_{m}(\sigma)>0 \text { for all } \sigma \in(0, \omega)
$$

hence, the definition of e-increasing operator is satisfied with $e(a) \equiv 1$

$$
\begin{aligned}
& \alpha\left(\psi_{1}, \psi_{2}\right)=B\left(e^{-\left\|v_{0}\right\|_{1}} e^{-\gamma \omega}-\left\|v_{0}\right\|_{1}\right) \int_{0}^{\omega}\left(\psi_{2}(\sigma)-\psi_{1}(\sigma)\right) \phi_{m}(\sigma) d \sigma \\
& \beta\left(\psi_{1}, \psi_{2}\right)=2 \max \left\{\left\|\Phi \psi_{1}\right\|_{\infty},\left\|\Phi \psi_{2}\right\|_{\infty}\right\} .
\end{aligned}
$$

Combining Lemma 11 and Proposition 12, we obtain

Theorem 13. Let $\rho(T)<1$ and assumptions 1-2 hold. Then, for $\left\|I_{y}\right\|_{1}$ small enough, there exists a unique equilibrium of (6). Moreover, the corresponding force of infection $\lambda^{*}$ satisfies $\lambda^{*} \leq(I-T)^{-1} u_{0}$.

Proof. Lemma 11 shows that all solutions lie in $\left[0,(I-T)^{-1} u_{0}\right]$, while Proposition 12 shows that, if

$$
\left\|(I-T)^{-1} u_{0}\right\| e^{\left\|(I-T)^{-1} u_{0}\right\|}<e^{-\gamma \omega},
$$

$\Phi$ is e-increasing on $\left[0,(I-T)^{-1} u_{0}\right]$.

Finally, when $\left\|I_{y}\right\|_{1}$ is small enough, (37) is satisfied (remember the definition (14) of $u_{0}$ ), and Theorem $\mathrm{D}$ can be applied.

Probably a sharper condition for $\Phi$ to be an increasing operator on $[0,(I-$ $\left.T)^{-1} u_{0}\right]$ can be found, but (37) suffices for our aims.

\section{A NUMERICAL EXAMPLE}

We illustrate our model by an example somehow inspired by the Italian demographic setting, and with epidemic parameters similar to what generally used for measles.

Precisely, fertility and mortality rates are taken from females' demographic data of Italy in 2004 [14]. In Fig. 1 we show the functions $m(\cdot)$ and $\Pi(\cdot)$ used. The corresponding value of $R$ is approximately 0.6 , so that it is definitely a belowreplacement population.

The age-dependent immigration rate has been parametrized as

$$
I_{x}(a)=I i_{x}(a) \quad I_{y}(a)=I i_{y}(a) \quad I_{z}(a)=I i_{z}(a)
$$




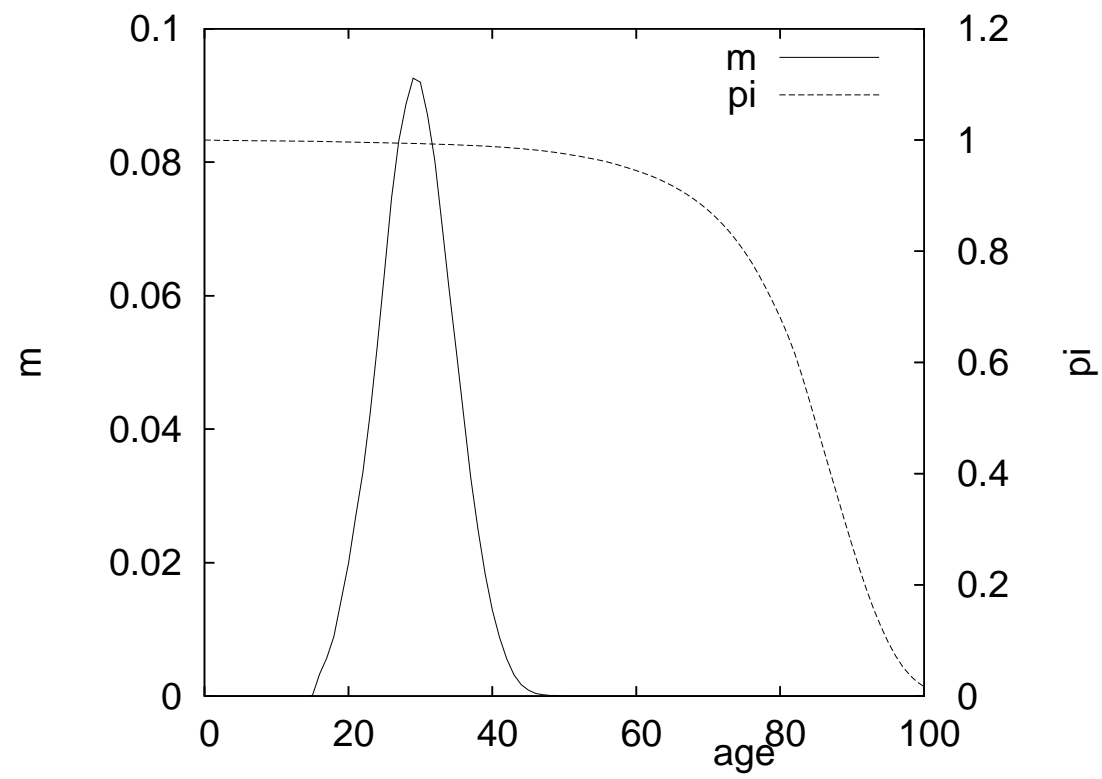

Figure 1 . The fertility $m(\cdot)$ and survival $\Pi(\cdot)$ functions used in the simulations.

where $i(a)=i_{x}(a)+i_{y}(a)+i_{z}(a)$ is such that $\int_{0}^{\omega} i(a) d a=1$. Hence, $I$ represents the total immigration rate, and will be used as a free parameter, while the function $i$ (a) represents the age profile of immigration. As the age profile of immigration we have used the profile reported in in Fig. 2, that was fitted by Manfredi and Valentini [19] to Italian immigration data prior to 2000. The profile shows the typical features of work migrations with a marked peak at young workers ages, plus a smaller peak at zero age.

From the demographic parameters and the immigration rate, one can compute through (2) the stationary population. Assuming $I=100,000$ (time is measured in years), we obtain the population shown in Fig. 2; total population is slightly above 9,500,000 inhabitants. Note however that the demographic rates consider only females, so that, assuming $1: 1$ sex ratio and equal survival of males and females, the total population would be around 19 millions.

As for the distribution of the immigrants among the epidemic classes, we have chosen

$$
i_{x}(a)=i(a) e^{-\lambda_{i} a} \quad i_{y}(a)=i(a) \frac{\lambda_{i}}{\gamma-\lambda_{i}}\left(e^{-\lambda_{i} a}-e^{-\gamma a}\right)
$$

with $\lambda_{i}=4(\text { year })^{-1}$ and $\gamma=52(\text { year })^{-1}$; clearly $i_{z}(a)=i(a)-i_{x}(a)-i_{y}(a)$. This amounts to assume that the source population (i.e the population where the migrants come from) is homogeneously mixing by age, with an average age at infection around 4 years, and moreover that migrants are not selected from an epidemioloigcal point of view. Since $\gamma$ is the recovery rate, this corresponds to an average infectious period of 1 week.

The contact rates are assumed to follow a WAIFW matrix, with values tailored to the dynamics of measles in Italy [18].

Precisely, we assume that

$$
\beta(a, s)=\sum_{i, j=1, \ldots, n} \beta_{i j} \chi_{I_{i}}(a) \chi_{I_{j}}(s)=\beta_{i j}, \quad \text { if } a \in I_{i}, s \in I_{j}, \quad i, j=1 \ldots n
$$




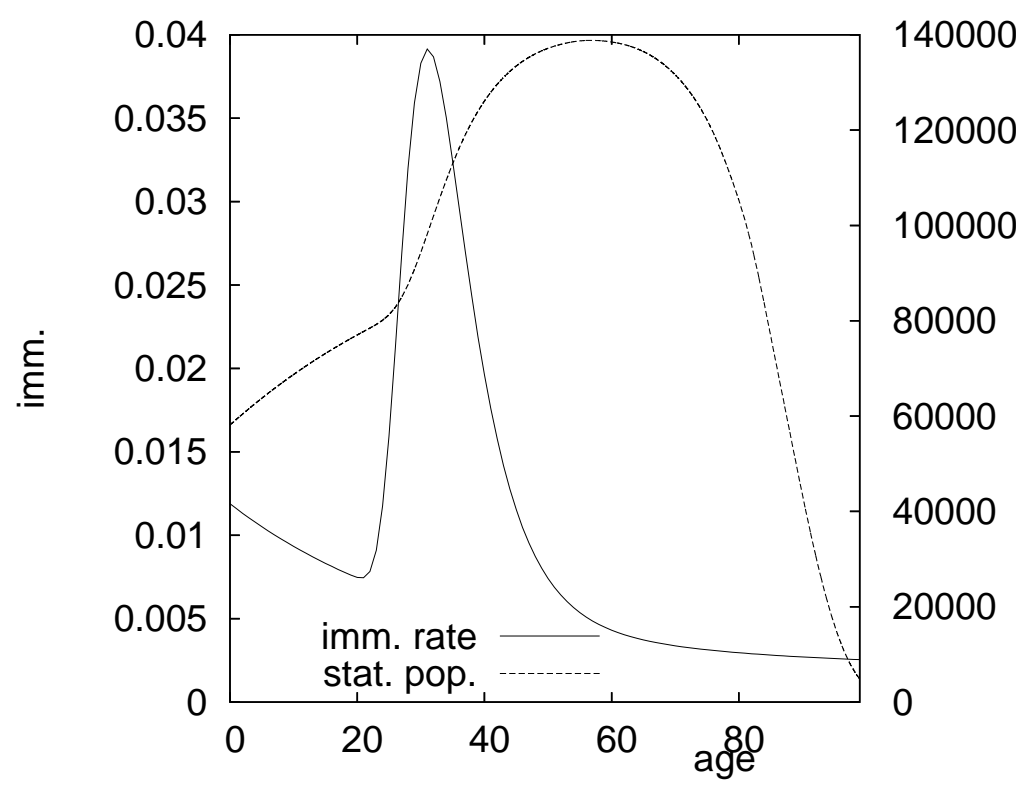

요

FIGURE 2. The immigration function used in the simulations, and the resulting stationary population. Age-dependent immigration rate $I(a)=I i(a)$, where $I=100,000$ and $i(\cdot)$ is the function shown in the figure. The stationary population is obtained with $I(a)$ and the functions $m(\cdot)$ and $\Pi(\cdot)$ shown in Fig. 1.

where $I_{i}=\left[a_{i-1}, a_{i}\right]$ are intervals partitioning $[0, \omega]$, i.e. $0=a_{0}<a_{1}<\ldots a_{n}=\omega$. In this case, we have

$$
(T \psi)(a)=\sum_{i} \chi_{I_{i}}(a) \int_{0}^{\omega} \psi(\sigma) n(\sigma) g_{i}(\sigma) d \sigma
$$

where

and

$$
n(\sigma)=B \pi(\sigma)+\int_{0}^{\sigma} I_{x}(s) \frac{\pi(\sigma)}{\pi(s)} d s
$$

$$
g_{i}(\sigma)=\sum_{j} \beta_{i j} \int_{\sigma}^{\omega} \chi_{I_{j}}(\xi) e^{-\gamma(\xi-\sigma)} \frac{\pi(\xi)}{\pi(\sigma)} d \xi
$$

so that the range of $T$ is in the subspace, $V_{n}$, generated by $\left\{\chi_{I_{i}}(\cdot), i=1, \ldots n\right\}$.

Hence, the eigenvalues of $T$ (and so its spectral radius) can be found by looking at the finite-dimensional operator $T: V_{n} \rightarrow V_{n}$ that (after some computations) can be represented by the matrix

$$
T_{i j}=\int_{a_{j-1}}^{a_{j}} k(\sigma)\left[\beta_{i j} Q_{j}(\sigma)+\sum_{l>j} \beta_{i l} Q_{l}\left(a_{l-1}\right) e^{-\gamma\left(a_{l-1}-\sigma\right)}\right] .
$$

where

$$
\begin{gathered}
k(\sigma)=B+\int_{0}^{\sigma} \frac{I_{x}(s)}{\pi(s)} d s, \sigma \in(0, \omega), \\
Q_{j}(\sigma)=\int_{\sigma}^{a_{j}} e^{-\gamma(\xi-\sigma)} \pi(\xi) d \xi, \sigma \in\left(a_{j-1}, a_{j}\right), \quad j=1 \ldots n .
\end{gathered}
$$

In the example, we chose $n=5$ and the age grouping $0-2,3-5,6-10,11-19,19+$ corresponding to main school grades in Italy. As regards the matrix $\beta$ the following 
structure used in epidemiological studies of the transmission dynamics of measles in Italy [21, 20] was adopted

$$
\left(\begin{array}{lllll}
\beta_{1} & \beta_{1} & \beta_{1} & \beta_{1} & \beta_{5} \\
\beta_{1} & \beta_{2} & \beta_{4} & \beta_{4} & \beta_{5} \\
\beta_{1} & \beta_{4} & \beta_{3} & \beta_{4} & \beta_{5} \\
\beta_{1} & \beta_{4} & \beta_{4} & \beta_{3} & \beta_{5} \\
\beta_{5} & \beta_{5} & \beta_{5} & \beta_{5} & \beta_{5}
\end{array}\right)
$$

so that, through (41), we are reduced to the computation of the spectral radius of a $5 \times 5$ matrix.

If (38) holds, it is easy to see that all terms of the matrix $T$ are linear in the parameter $I$ (remember the definition (3) of $B$ ), so that the spectral radius of $T$ is a linear function of $I$. It is then easy to find the threshold value of $I$, i.e. the value at which $\rho(T)=1$ that, with the numerical values specified in Fig. 3 , is $I \approx 74.6$.

In Fig. 3 we show the equilibrium values of the proportion infected, $Y / N$, vs. the value of the immigration rate. It can be seen that, below the threshold $(I<74.6)$, the proportion infected is very close to 0 , while above the threshold it rises sharply. As can be seen from (3)-(2), the population density at demographic equilibrium

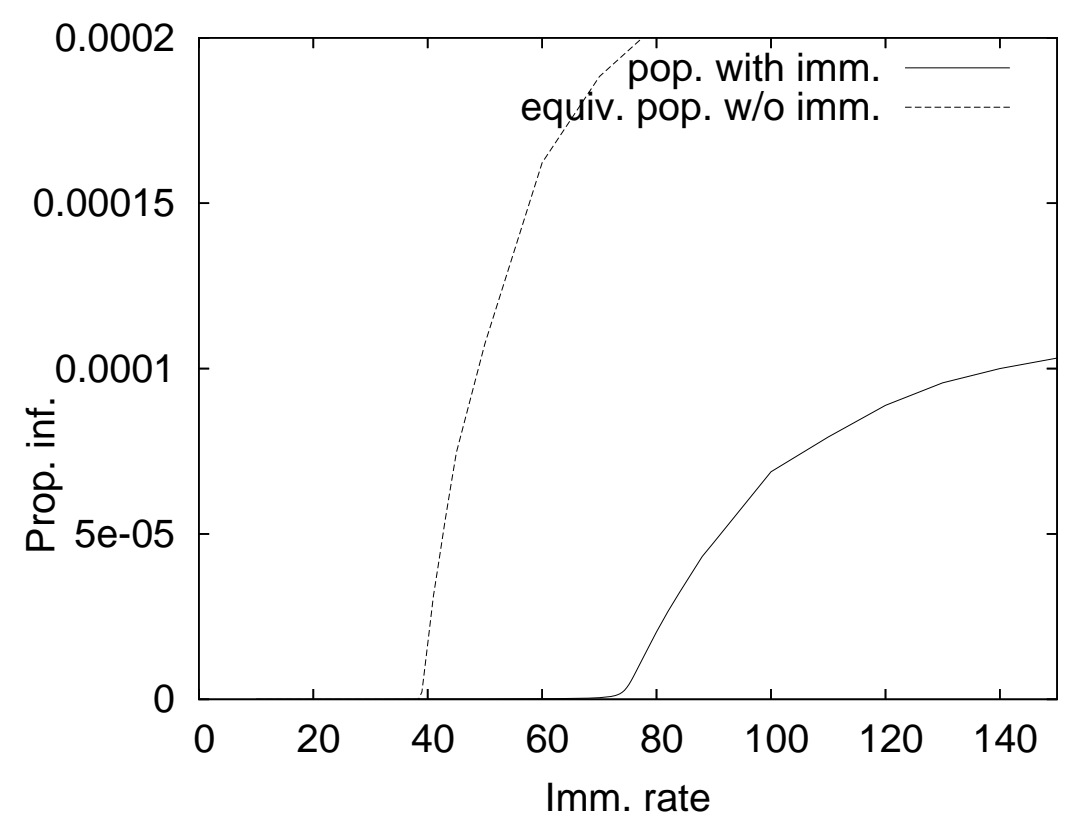

Figure 3. The solid line shows the proportion infected, i.e. $\frac{\int \bar{Y}(a) d a}{\int n(a) d a}$, at the equilibrium value of system (6) for different values of total immigration rate $I$. $\Pi(\cdot)$ as in Fig. 1, relative immigration rate as in Fig. 2. The contact rate has the structure (40) with the matrix $\beta$ given by (42). The dashed line shows the proportion infected in a population with the same population densities, mortalities and contact rates, but without immigration. Parameter values are $\beta_{1}=1.254 \cdot 10^{-5}, \beta_{2}=4.68 \cdot 10^{-5}, \beta_{3}=1.16 \cdot 10^{-4}$, $\beta_{4}=1.986 \cdot 10^{-5}, \beta_{5}=1.042 \cdot 10^{-5}$; the ages used in $(40)$ are $a_{1}=3, a_{2}=6, a_{3}=11, a_{4}=19 ; \gamma=52$.

is an increasing function of total immigration. Thus, since the force of infection increases linearly with population density, the infection prevalence increases with 
$I$, as shown in Fig. 3. However, the joint action of BRF and immigration yields a stationary population density which is significantly older compared to an underlying stationary through births and deaths only population, as can be seen in Fig. 2.

In order to disentangle the effect of age structure from that of overall population density, we considered a stationary population without immigration, that has however the same population density (and the same mortality, but higher fertilities) as the population with immigration studied here. The dashed line in Fig. 3 shows, for each immigration level $I$, the equilibrium infection prevalence in a population without immigration with the same population density as the population with immigration level $I$.

Comparing the solid and dashed line in Fig. 3, one immediately sees that a BRF population maintained by immigration has a much lower infection prevalence (and a higher threshold for persistence) than a population where stationarity follows from the balance of births and deaths only. This is because the former population has a much older age structure that is a much smaller fraction of young individuals (and in our example contact rates are generally higher among younger age-classes and most immigrants will have already been naturally immunized in their countries).

Looking carefully, one can also see a qualitative difference between the shapes of the solid and dashed lines. The dashed line shows a sharp threshold, with no infection below the threshold. On the other hand, one may note that with immigration (the solid line) the proportion of infected individuals, while displaying a sharp bend at the threshold, grows steadily also below the threshold (we have a 'quasi-threshold'). If we had chosen a smaller proportion of infective immigrants, the curve would look sharper, as Theorem 3 states.

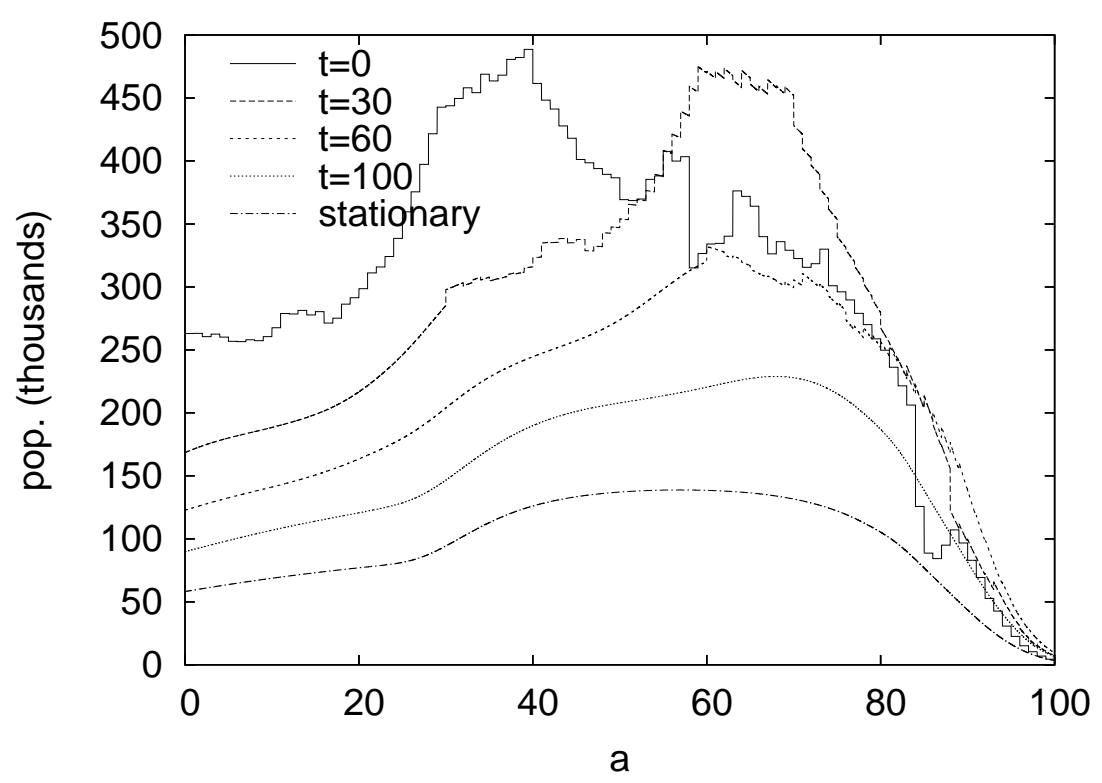

FigurE 4. The age distribution of the population with constant fertility, mortality and immigration rates at different times. See the text for more explanation.

Finally, we analyse through simulations the importance of transients for the model. To this purpose, we consider again system (6a), but with initial conditions based on the current Italian demographic structure so that $n(a, t)=X(a, t)+$ 
$Y(a, t)+Z(a, t)$ is not constant in time; hence, we also changed the boundary condition $(6 \mathrm{~b})$ to

$$
X(0, t)=\int_{0}^{\omega} m(a) n(a, t) \quad Y(0, t)=Z(0, t)=0 .
$$

In Fig. 4, we show the predicted demographic evolution starting from the current Italian demographic structure with constant fertility and mortality rates shown in Fig. 1, and immigration rate shown in Fig. 2.

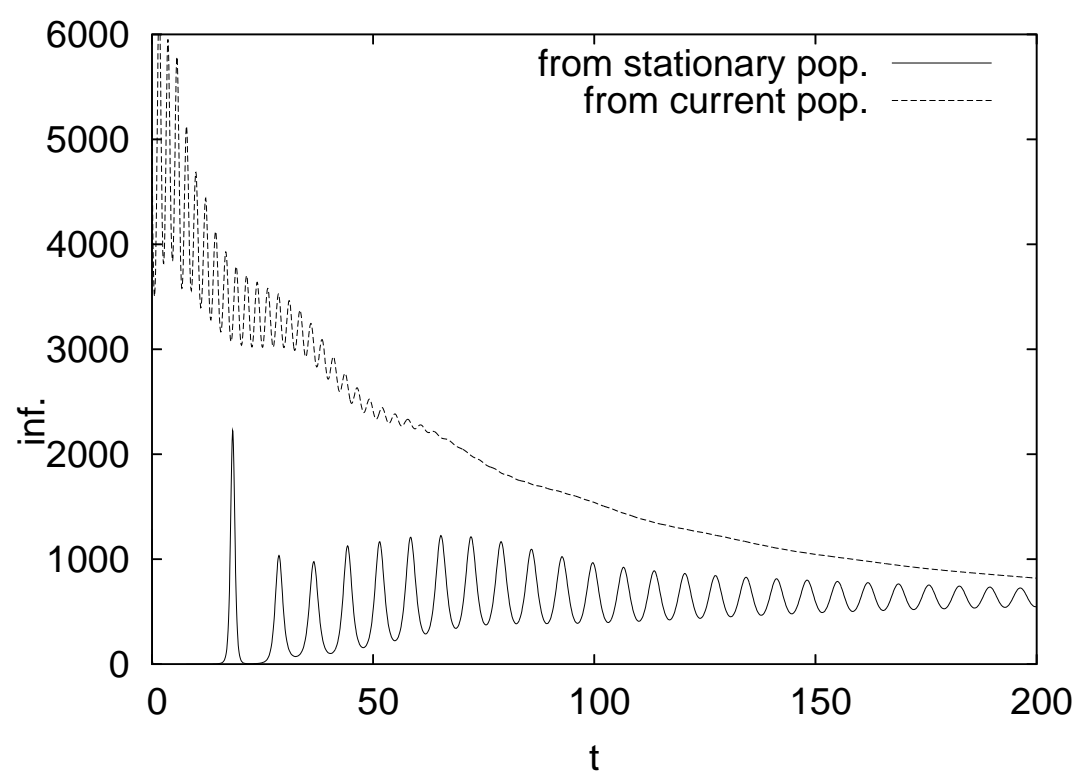

Figure 5. Total number of infectives vs. time. The solid line shows the solution of (6) starting from the stationary population (3)-(2); the dashed line the solution starting from the population shown, at $t=0$, in Fig. 4. Parameter values are as in Fig. 3 with $I=100,000$.

In Fig. 5, we show the predicted evolution of the total number of infectives under two different scenarios: in the first, we start from an initial population inspired by the current demographic situation, as outlined above; in the second, we start from the stationary population shown in Fig. 2; in both simulations the initial fractions of susceptibles, infectives and immune are close to what would be a stationary solution consistent with the force of infection estimated for measles in the Italian population [18]. It can be seen that the two simulations are rather different: starting from the current demographic situation, initially there are oscillations, close to a biennial period, in the number of infectives, while starting from the stationary population one needs (because the overall population density is lower) several years for the susceptibles to build up enough to sustain an epidemic, followed by longer and milder oscillations. Further on, however, the oscillations in the solution "from current" damp out quickly, and, after a few decades, the number of infectives decreases steadily towards the equilibrium; on the other hand, the oscillations in the solution "from stationary" persist over all 200 years of simulations. It apppears then that the demographic transient has a strong stabilizing effect on the epidemic oscillations, and this fact, not easily explained, has been verified with several other initial conditions. 
Moreover, the influence of initial conditions can still be seen after 200 years of simulations, since the two solutions are still noticeably different. Hence, the analysis of the stationary case, while it may emphasize relevant quantities to consider, is not an adequate predictor of the dynamics of the infection over a reasonable time horizon.

\section{Acknowledgements}

We are strongly indebted, for this work, to Piero Manfredi, that has suggested the relevance of the problem, has introduced us to the demographic literature, and has made several comments and suggestions on previous drafts of this paper.

\section{REFERENCES}

[1] H. Amann. On the number of solutions of nonlinear equations in ordered Banach spaces. Journal of Functional Analysis, 11:346-384, 1972.

[2] R.M. Anderson and R.M. May. Vaccination against rubella and measles: quantitative investigations of different policies. J. Hyg. Camb, 90:259-325, 1983.

[3] Roy M. Anderson and Robert M. May. Infectious diseases of humans: dynamics and control. Oxford University Press, Oxford New York Tokio, 1991.

[4] W.B. Arthur, T.J. Espenshade, and L.J. Bouvier. Immigration and the stable population model. Demography, 19:125-133, 1982.

[5] F. Brauer and P.van den Driessche. Models for transmission of disease with immigration of infectives. Mathematical Biosciences, 171:143-154, 2001.

[6] S. Busenberg, K. Cooke, and M. Iannelli. Endemic thresholds and stability in a class of age-structured epidemics. SIAM J. Appl. Math., 48:1379-1395, 1988.

[7] P. Cerone. On stable population theory with immigrations. Demography, 24/3:431-438, 1987.

[8] D. Greenhalgh. Analytical results on the stability of age-structured recurrent epidemic models. IMA Journal of Mathematics applied in Medicine and Biology, 4:109-144, 1987.

[9] D. Greenhalgh. Threshold and stability results for an epidemic model with an age structured meeting-rate. IMA Journal of Mathematics applied in Medicine and Biology, 5:81-100, 1988.

[10] H.W. Hethcote. Asymptotic behaviour and stability in epidemic models. In P. van den Driessche, editor, Mathematical Problems in Biology, volume 2 of Lectures Notes in Biomathematics, pages 83-92. Springer, Berlin Heidelberg New York, 1974.

[11] M. Iannelli and P. Manfredi. Demographic change and immigration in age-structured epidemic models. Mathematical Population Studies, to appear, 2007.

[12] H. Inaba. Asymptotic properties of the Inhomogeneous Lotka- Von Foerster System. Mathematical Population Studies, 1:257-264, 1988.

[13] H. Inaba. Threshold and stability results for an age-structured epidemic model. Journal of mathematical biology, 28:411-434, 1990.

[14] Italian National Institute of Statistics. Demographic data. http://demo.istat.it/, 2006.

[15] T. Kato. Perturbation theory for linear operators. Springer-Verlag, Berlin Heidelberg New York Tokio, 1966.

[16] H.P. Kohler, F.C. Billari, and J.A. Ortega. The emergence of lowest-low fertility in europe during the 1990s. Population. E Development Review , 4:641-680, 2002.

[17] M.G. Krein and M.A. Rutman. Linear operators leaving invariant a cone in a Banach space. AMS translations, 10:199-325, 1950.

[18] P. Manfredi, E.M. Cleur, J.R. Williams, S. Salmaso, and M. Ciofi dagli Atti. The prevaccination regional epidemiological landscape of measles in Italy: contact patterns, effort needed for eradication, and comparison with other regions of Europe. Population Health Metrics, 3(1), 2005.

[19] P. Manfredi and A. Valentini. Populations with below replacement fertility and immigration: theoretical considerations and scenarios from the 'Italian laboratory'. Riv. Int. Sci. Soc., $108(1): 61,2000$.

[20] P. Manfredi and J.R. Williams. Realistic population dynamics in epidemiological models: the impact of population decline on the dynamics of childhood infectious diseases. Measles as an example. Mathematical Biosciences, 192:153-175, 2004.

[21] P. Manfredi, J.R. Williams, S. Salmaso, and M. Ciofi dagli Atti. Measles elimination in Italy: the projected impact of the National Elimination Plan. Epidemiology \& Infection, 134(1), 2005.

[22] I. Marek. Frobenius theory of positive operators: comparison theorems and applications. SIAM J. Appl.Math., 19:607-628, 1970. 
[23] H.H. Schaefer. Banach Lattices and Positive Operators. Springer-Verlag, Berlin Heidelberg New York Tokio, 1974.

[24] D. Schentzle. An age structured model of pre- and post-vaccination measles transmission. IMA J.Math.Appl.Med.Biol., 1:169-191, 1984.

[25] United Nations. Replacement migration: is it a solution to declining and ageing populations?, 2002.

Dipartimento di Matematica, Università di Trento, Via Sommarive 14, 38050 Povo (TN), ITALY

Current address: Dipartimento di Statistica e Matematica applicata all'Economia, Via Cosimo Ridolfi 10, 56124 Pisa, Italy

E-mail address: francesc@ec.unipi.it

Dipartimento di Matematica, Università di Trento, Via Sommarive 14, 38050 Povo (TN), ITALY

E-mail address: pugliese@science.unitn.it 